\title{
Obesity-associated microRNA-26b regulates the proliferation of human preadipocytes via arrest of the G1/S transition
}

\author{
GUANGFENG XU ${ }^{1 *}$, CHENBO JI $^{2 *}$, GUIXIAN SONG ${ }^{3}$, CHUNMEI SHI $^{4}$, YAHUI SHEN ${ }^{2}$, \\ LING CHEN $^{4}$, LEI YANG ${ }^{4}$, YAPING ZHAO ${ }^{1}$ and XIRONG GUO ${ }^{2}$ \\ ${ }^{1}$ Department of Laboratory Medicine, The 82nd Hospital of The People's Liberation Army, Huaian, Jiangsu 223001; \\ ${ }^{2}$ Department of Child Health Care, Affiliated Nanjing Maternity and Child Health Care Hospital, Nanjing Medical University; \\ ${ }^{3}$ Department of Cardiology, The First Affiliated Hospital of Nanjing Medical University; ${ }^{4}$ Institute of Pediatrics, \\ Nanjing Medical University, Nanjing, Jiangsu 210029, P.R. China
}

Received July 27, 2014; Accepted April 10, 2015

DOI: $10.3892 / \mathrm{mmr} .2015 .3858$

\begin{abstract}
MicroRNAs (miRNAs) are short, 20-24 nucleotide non-coding RNAs, which are involved in multiple biological processes, including obesity. Our previous investigation revealed that miRNA (miR)-26b is differentially expressed in preadipocytes and mature adipocytes in humans. However, its role in the proliferation of human preadipocytes remains to be fully elucidated. In the present study, intracellular lipid accumulation was assessed using oil red $\mathrm{O}$ staining and the trigycerlide (TG) content was quantified using a TG assay kit, adipogenesis associated genes and cyclin D2 were analyzed using western blotting, and the effects of miR-26b on the proliferation of preadipocytes was investigated using Cell Counting Kit-8 assays and cell cycle analysis. Human preadipocytes overexpressing miR-26b exhibited increased TG content in the adipocytes. During differentiation, the protein expression levels of adipogenesis-associated marker genes, including peroxisome proliferator-activated receptor $\gamma$, CCAAT/enhancer-binding protein $\alpha$, fatty acid-binding protein and hormone-sensitive lipase were upregulated in cells overexpressing miR-26b, compared with the negative control cells. In addition, growth of human preadipocytes overexpressing miR-26b occurred a slower rate and more remained in the G1 phase, compared with the negative control cells. In addition,
\end{abstract}

Correspondence to: Ms. Yaping Zhao, Department of Laboratory Medicine, The 82nd Hospital of The People's Liberation Army, 100 Jiankang East Road, Huaian, Jiangsu 223001, P.R. China

E-mail: zyp82yy@aliyun.com

Professor Xirong Guo, Department of Child Health Care, Affiliated Nanjing Maternity and Child Health Care Hospital, Nanjing Medical University, 123 Tianfei Road, Nanjing, Jiangsu 210029, P.R. China E-mail: xrguo@njmu.edu.cn

*Contributed equally

Key words: preadipocytes, proliferation, differentiation, microRNA, microRNA-26b
miR-26b downregulated the protein expression of cyclin D2. These results demonstrated that miR-26b promoted differentiation and, at least party by targeting cyclin D2, attenuated cell proliferation via arresting the G1/S transition.

\section{Introduction}

Obesity and obesity-associated diseases have reached pandemic levels and are prevalent even in developing countries. It is of concern that the prevalence of obesity among children and adolescents is also increasing $(1,2)$. Obesity is the excessive accumulation of adipose tissue due to energy imbalance. Adipocytes represent the units of adipose tissue and are the basis of adipose tissue functionality. Obesity is characterized by an increase in the number and volume of adipocytes, and is associated with adipocyte differentiation and proliferation $(3,4)$. Therefore, elucidation of the molecular mechanisms governing adipocyte differentiation and proliferation is important in preventing obesity and its sequelae.

MicroRNAs (miRNAs) are a novel class of endogenous, non-coding RNAs, 20-24 nucleotides in length, which are important in several physiological and pathological processes, including cell growth, differentiation, and development (5). miRNAs bind to the 3' untranslated regions (UTR) of target mRNAs, and either repress their translation and/or promote their decay (6). The expression of miRNAs in the proliferation and differentiation of murine adipocytes has been investigated, however, the involvement of miRNAs in human adipocyte proliferation and differentiation remains to be fully elucidated. miR-21 has been reported to regulate the proliferation of human adipose tissue-derived mesenchymal stem cells (7). In addition, a primate-specific microRNA (miR-637) suppresses the growth of human mesenchymal stem cells and induces $\mathrm{S}$ phase arrest (8). In human preadipocytes, Esau et al (9) demonstrated that miR-143 is induced during differentiation, and its inhibition with antisense oligonucleotides inhibited preadipocyte differentiation. Karbiener et al (10) reported that the expression of miR-30c increases during adipogenesis of human multipotent adipose-derived stem cells, and its overexpression induces adipocyte marker gene induction and triglyceride accumulation. Additionally, Lee et al (11) found 
that overexpressing miR-130 impairs adipogenesis and reduces miR-130-enhanced adipogenesis. These findings indicate that miRNAs may be prominent in regulating the proliferation and differentiation of human adipocytes.

In the present study, the role of miR-26b in the differentiation and proliferation of human preadipocytes was assessed, by comparing human preadipocytes overexpressing miR-26b with negative control cells.

\section{Materials and methods}

Cell culture and differentiation. Human preadipocytes (HPA-V) (ScienCell Research Laboratories, San Diego, CA, USA) were maintained in preadipocyte medium (PAM; ScienCell Research Laboratories, San Diego, CA, USA) supplemented with 5\% fetal bovine serum (FBS; Gibco Life Technologies, Carlsbad, CA, USA), $1 \%$ preadipocyte growth supplement (PAGS; ScienCell Research Laboratories), and $1 \%$ penicillin/streptomycin solution (P/S; ScienCell Research Laboratories) at $37^{\circ} \mathrm{C}$ in a humidified atmosphere with $5 \% \mathrm{CO}_{2}$. In order to induce differentiation, fully confluent human preadipocytes (day 0) were exposed to a differentiation cocktail (Sigma-Aldrich, St. Louis, MO, USA) of serum-free PAM, containing $50 \mathrm{nM}$ insulin, $100 \mathrm{nM}$ dexamethasone, $0.5 \mathrm{mM}$ 3-isobutyl-1-methylxanthine and $100 \mu \mathrm{M}$ rosiglitazone. The culture medium was replaced every 2 days for the first 4 days. Thereafter, the medium was replaced with serum-free PAM, containing $50 \mathrm{nM}$ insulin, which was replaced every 2 days until the accumulation of lipid droplets was observed (days 15-17).

miRNA minigene and lentivirus-mediated cell transduction. The pGLV-H1-green fluorescent protein (GFP)+Puro (pGLV3) plasmids used to construct the miR-26b minigene were obtained from GenePharma (Shanghai, China). The fragments, which contained the coding sequence of miR-26b (miR-26b minigene) were amplified by polymerase chain reaction (PCR), using the genomic DNA extracted from human adipocytes, and were ligated into the GFP 3'-UTR of the BamHI and EcoRI-digested pGLV3 vector. PCR reactions were carried out in a $25 \mu \mathrm{l}$ reaction volume containing $200 \mathrm{ng}$ DNA, $0.25 \mu \mathrm{l}$ 5U/ $\mu 1$ Takara Ex Taq HS (Takara Bio Inc., Otsu, Japan), $2.5 \mu 1$ 10X PCR buffer $\left(\mathrm{Mg}^{2+}\right)$, $2 \mu 12.5 \mathrm{mM}$ dNTP mixture, $0.25 \mu \mathrm{l}$ of each outer PCR primer (Invitrogen Life Technologies, Carlsbad, CA, USA); forward primer, 5'-ACCCTCGAGAGGCTCTTCCCACCAATCCG-3' and reverse primer, 5'-GCCGGATCCGGCCAGCTACCCTGA CCACT-3', and the mixture was made up to $25 \mu 1$ with $\mathrm{H}_{2} \mathrm{O}$. The PCR system used was Arktik ${ }^{\mathrm{TM}}$ Thermal Cyclers (Thermo Scientific Inc., Waltham, MA, USA) and the PCR conditions were as follows: $95^{\circ} \mathrm{C}$ for $5 \mathrm{~min}$, followed by 35 cycles at $94^{\circ} \mathrm{C}$ for $30 \mathrm{sec}, 55^{\circ} \mathrm{C}$ for $30 \mathrm{sec}$ and $72^{\circ} \mathrm{C}$ for $1 \mathrm{~min}$, and $72^{\circ} \mathrm{C}$ for $10 \mathrm{~min}$. The positive recombinant pGLV3 plasmids were identified and screened for using enzyme digestion and sequencing. To establish preadipocyte cells containing the miR-26b minigene, a lentivirus-mediated cell-transfection technique was used. Once the HEK-293T cells (American Type Culture Collection, Manassas, VA, USA) had grown to $60 \%$ confluence, they were co-transfected with the miR-26b minigene plasmids and packaging plasmids (pGag/Pol, pVSV-G and pRev; GenePharma) or empty plasmids, using Lipofectamine 2000 (Invitrogen Life Technologies) as a transfection reagent. The HEK-293T cells were cultured in Dulbecco's modified Eagle's medium (Wisent Inc., St. Bruno, QC, Canada), supplemented with 10\% FBS, in a $37^{\circ} \mathrm{C}$ incubator with $5 \% \mathrm{CO}_{2}$. The lentivector-containing supernatants were collected $72 \mathrm{~h}$ after transfection. The viral supernatants were centrifuged at $8,000 \mathrm{x} \mathrm{g}$ for $10 \mathrm{~min}$ at $4^{\circ} \mathrm{C}$ to remove cell debris, filtered through $0.45-\mu \mathrm{m}$ filters (Millipore, Billerica, MA, USA), supplemented with hexadimethrine bromide (polybrene; Sigma-Aldrich) to a final concentration of $5 \mu \mathrm{g} / \mathrm{ml}$, and applied directly to the preadipocyte cells (confluence, $\sim 60 \%$ ). Subsequently, fluorescence microscopy (Olympus BX43; Olympus Corporation, Tokyo, Japan) was used to observe the infection efficiency, via the expression of GFP, and the cells were harvested to determine the level of miR-26b overexpression 3 days post-infection.

Reverse transcription-quantitative PCR (RT-qPCR). Total RNA was extracted from the adipocytes using TRIzol (Invitrogen Life Technologies) and quantified spectrophotometrically (NanoDrop Products, Wilmington, DE, USA) at $260 \mathrm{~nm}$. Mature miRNA quantification was performed using TaqMan ${ }^{\circledR}$ miRNA arrays (Applied Biosystems, Foster City, CA, USA). The TaqMan MicroRNA Reverse Transcription kit (Applied Biosystems) was used to generate cDNA. The expression of miR-26b was analyzed using RT-qPCR in an Applied Biosystems 7500 Sequence Detection system (ABI 7500 SDS; Applied Biosystems). For PCR, $1 \mu \mathrm{l}$ cDNA (1:20 dilution), $0.1 \mu \mathrm{M}$ TaqMan probe, $0.2 \mu \mathrm{M}$ forward primer, $0.2 \mu \mathrm{M}$ reverse primer and TaqMan Universal PCR Master Mix II (Applied Biosystems) were included in a $20 \mu \mathrm{l}$ reaction mixture. The PCR conditions were as follows: $10 \mathrm{~min}$ at $95^{\circ} \mathrm{C}$, followed by 40 cycles at $95^{\circ} \mathrm{C}$ for $15 \mathrm{sec}$ and $60^{\circ} \mathrm{C}$ for $1 \mathrm{~min}$ then a final extension step of $72^{\circ} \mathrm{C}$ for $5 \mathrm{~min}$. The relative expression levels of miR-26b were quantified based on the cycle threshold $(\mathrm{Ct})$ values and were normalized to the snRU6 reference gene. Each sample was measured in triplicate, and the expression levels of miR-26b were calculated using the $2^{-\Delta \Delta \mathrm{Ct}}$ method, as described previously (12). $\Delta \Delta \mathrm{CT}=$ Avg. $(\mathrm{CTmiR}-26 \mathrm{~b}-\mathrm{CT}$ snRU6) - Avg . (CTNC-CTsnRU6).

Oil red $O$ staining. For oil red $\mathrm{O}$ staining, the medium was removed from the cell culture plates and the adipocytes were washed three times with PBS, followed by being fixed with $4 \%$ formalin in phosphate buffer (Sigma-Aldrich) for $30 \mathrm{~min}$ at room temperature. Following fixation, the cells were washed twice with PBS and stained with $0.6 \%(\mathrm{w} / \mathrm{v})$ filtered oil red O solution (Sigma-Aldrich) (60\% isopropanol, $40 \%$ water) for $30 \mathrm{~min}$ at room temperature. Subsequently, the cells were washed with tap water to remove any unbound dye, were visualized using light microscopy (Olympus BX43; Olympus Corporation) and images of the cells were captured.

Quantification of triglyceride content. The human preadipocytes were cultured in 6-well plates and treated to differentiate into adipocytes, as described above. The cells were then collected after 4,10 and 15 days. The cells were washed twice with $1 \mathrm{ml} \mathrm{PBS}$ (at $4^{\circ} \mathrm{C}$ ) and then dissolved in 200-400 $\mu 1$ 1\% Triton X-100 (Sigma-Aldrich) by sonication (B5500S-DTH; Branson Ultrasonics Corp., Shanghai, China). The whole-cell lysates were then centrifuged at 8,000 rpm for $5 \mathrm{~min}$ at $4^{\circ} \mathrm{C}$. The intracellular triglyceride (TG) content 
was measured using a TG assay kit (GPO-POD; Applygen Technologies Inc., Beijing, China), according to the manufacturer's instructions. The protein concentration was quantified using a Pierce BCA Protein Assay kit (Thermo Fisher Scientific Inc.), expressed as $\mu \mathrm{mol} \mathrm{TG} / \mathrm{mg}$ cellular protein.

Cell proliferation assay. The proliferation rates of the miR-26b minigene cells and the negative control cells were measured using a WST-8 Cell Counting Kit-(CCK)8 (Dojindo Molecular Technologies, Inc., Tokyo, Japan), according to the manufacturer's instructions. Briefly, the cells (2,000 cells/well) were seeded into 96-well plates and incubated in PAM, supplemented with 5\% FBS, $1 \%$ PAGS and 1\% P/S for 4 days. After $0,12,24$, 48,72 or $96 \mathrm{~h}$ of growth, $100 \mu \mathrm{l}$ of the media was replaced with an equal volume of fresh media containing 10\% CCK-8, and the cultures were incubated at $37^{\circ} \mathrm{C}$ for $60 \mathrm{~min}$. The absorbance was measured at an optical density (OD) of $450 \mathrm{~nm}$ using a Multiskan MK3 microplate reader (Thermo Fisher Scientific Inc.). The difference between these values was recorded as the OD value. The results are expressed as the mean \pm standard deviation(SD) of three separate experiments, with six determinations per experiment for each experimental condition.

Cell cycle assay. The cell cycle distribution was analyzed using flow cytometry. The cells $\left(2 \times 10^{6} / 75 \mathrm{~cm}^{2}\right)$ were cultured in PAM with 5\% FBS, $1 \%$ PAGS and 1\% P/S. Following adhesion, the cells were washed twice with PBS and starved in serum-free PAM for $24 \mathrm{~h}$. Following replacement of the starvation medium with complete medium, cell cycle analysis was performed at 0,24 and $48 \mathrm{~h}$. The cultured cells were harvested using trypsin/EDTA (Sigma-Aldrich) and washed twice with PBS. Aliquots of at least $2 \times 10^{6}$ cells were centrifuged, fixed in $75 \%$ ethanol (Sigma-Aldrich) and stained with $500 \mu \mathrm{l}$ propidium iodide (PI) solution (Sigma-Aldrich), containing $100 \mu \mathrm{g} / \mathrm{ml}$ RNase (Sigma-Aldrich) and $50 \mu \mathrm{g} / \mathrm{ml}$ PI in 1X PBS. The labeled cells were analyzed on a BD FACScan (BD Biosciences, Franklin Lakes, NJ, USA) and data were analyzed using CellQuest version 5.1 software (BD Biosciences).

Western blotting. Human preadipocytes were lysed in immunoprecipitation assay buffer (Beyotime Institute of Biotechnology, Shanghai, China), containing $150 \mathrm{mM} \mathrm{NaCl}, 1.0 \%$ IGEPAL CA-630, $0.5 \%$ sodium deoxycholate, $0.1 \%$ SDS and $50 \mathrm{mM}$ Tris ( $\mathrm{pH} 8.0$ ). The protein concentrations were determined using the BCA protein assay kit (Thermo Fisher Scientific Inc.). The proteins were separated on a $10 \%$ SDS/PAGE gel (Thermo Fisher Scientific Inc.) under reducing conditions (containing 2-mercaptoethanol) and were electroblotted onto a polyvinylidine difluoride membrane (Beyotime Institute of Biotechnology). The membranes were probed using primary antibodies against cyclin D2 (rabbit polyclonal; 1:1,000; cat. no. 2924S; Cell Signaling Technology, Danvers, MA, USA) and $\beta$-actin (mouse monoclonal; $1: 1,000$; cat. no. A5316; Sigma-Aldrich). The membranes were blocked with 5\% nonfat milk (Bio-Rad Laboratories, Inc., Hercules, CA, USA) for $1 \mathrm{~h}$ at room temperature and probed with primary antibodies at $4^{\circ} \mathrm{C}$ overnight. The membranes were washed 3 times with Tris-buffered saline with $0.05 \%$ Tween-20 (Beyotime Institute of Biotechnology) and incubated with horseradish peroxidase-conjugated anti-rabbit or mouse $\operatorname{IgG}(1: 5,000)$ for
A

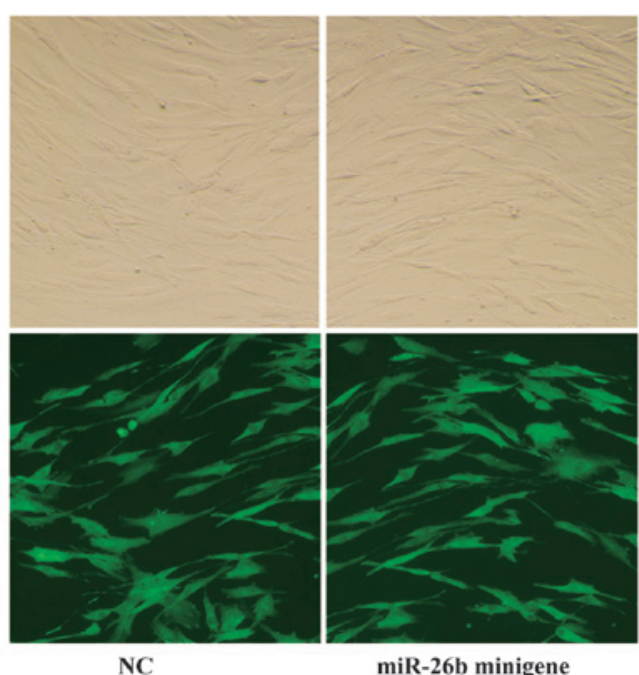

B

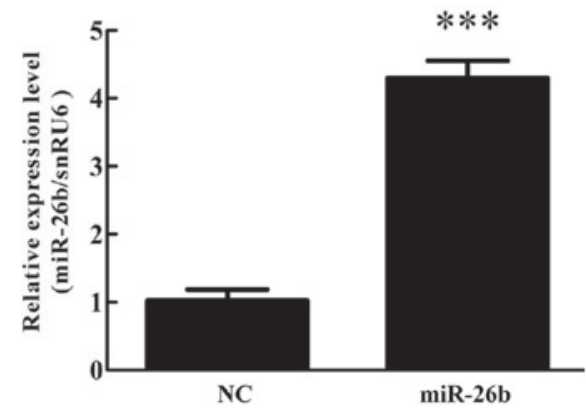

Figure 1. Validation of miR-26b overexpression. Fluorescence microscopy (magnification, x200) of human preadipocytes was used to determine the transduction efficiency via the expression of green fluorescent protein. (A) miRNA isolated from lentivirus-transduced human preadipocytes was subjected to reverse transcription-quantitative polymerase chain reaction. (B) In each sample, the data were normalized to the level of snRU6. The results are presented as the mean \pm standard deviation of three experiments $\left({ }^{* * *} \mathrm{P}<0.001\right.$, vs. NC). NC, negative control; miR, microRNA; snRU6, U6 small nucleolar RNA.

$1 \mathrm{~h}$ at room temperature. Signals were detected using enhanced chemiluminescence (Perkin-Elmer Inc., Waltham, MA, USA) and band intensities were quantified using Image J V1.47.

Statistical analysis. Each experiment was performed at least three times. Data are presented as the mean \pm SD. Statistical analyses were performed using paired Student's t-test, with the SPSS 11.0 statistical software package (SPSS Inc., Chicago, IL, USA). $\mathrm{P}<0.05$ were considered to indicate a statistically significant difference.

\section{Results}

Confirmation of miR-26b overexpression. The present study used a lentivirus overexpressing miR-26b, the miR-26b minigene, in human preadipocytes. The lentivirus vector contained the GFP-coding sequence, the expression of which is driven by a cytomegalovirus promoter. Therefore, lentivirus transduction efficiency can be determined using a fluorescent microscope. Transduction with the miR-26b minigene or 
$\mathbf{A}$

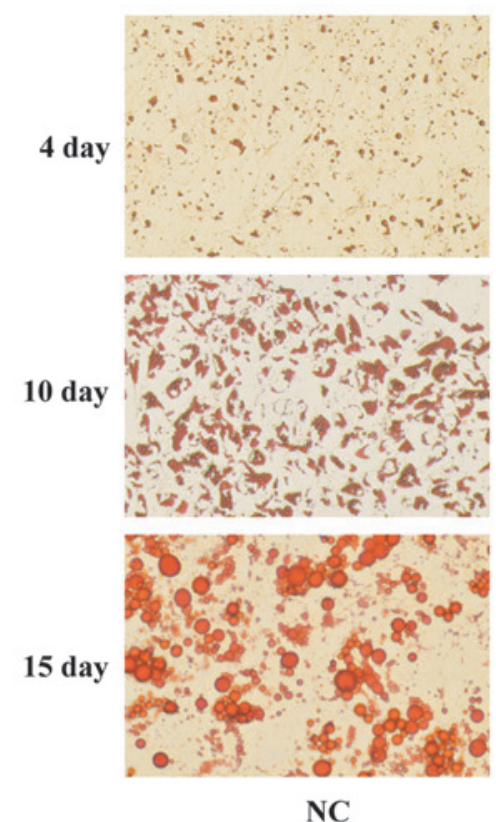

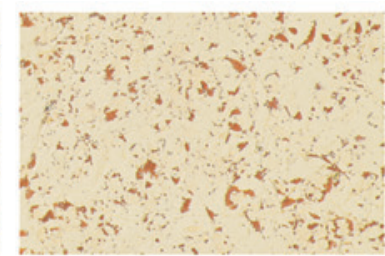
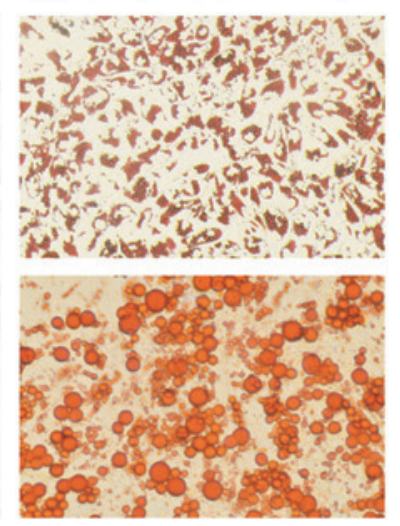

miR-26b minigene
B

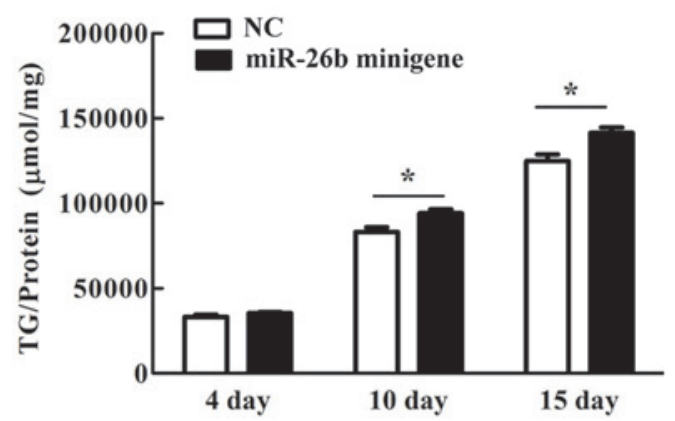

Figure 2. Effect of miR-26b on lipid accumulation and lipid droplet formation in human preadipocytes. Human preadipocytes were infected with miR-26b minigene or negative control lentivirus and were stimulated to differentiate over 15 days. (A) On days 4,10 and 15, images of the morphological changes associated with cell differentiation were captured following oil red O staining (magnification, x200). (B) Cell triglyceride content was measured using a triglyceride assay kit. Data are expressed as the mean \pm standard deviation $\left(\mathrm{n}=6 ;{ }^{*} \mathrm{P}<0.05\right)$. NC, negative control; miR, microRNA; TG, triglyceride.

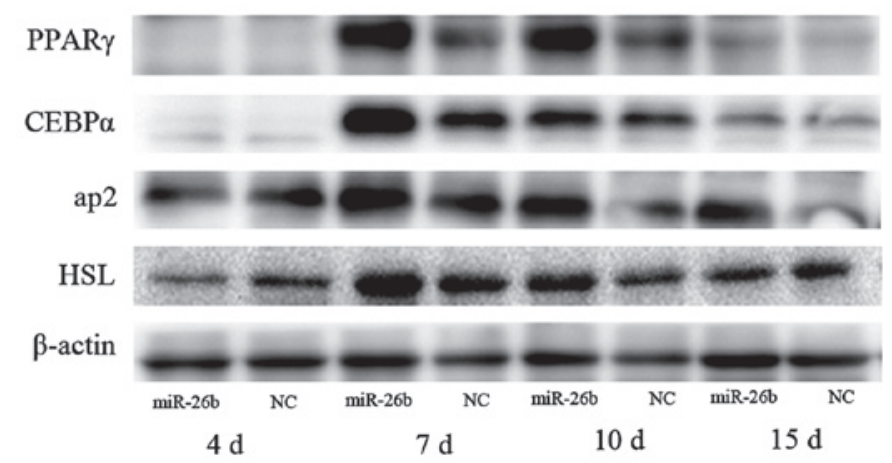

Figure 3. Expression of adipogenesis markers during cell differentiation in miR-26b minigene-transfected preadipocytes. Human preadipocytes were induced to differentiate and the protein levels of PPAR $\gamma, \mathrm{CEBP} \alpha$, aP2 and HSL, normalized to $\beta$-actin, were analyzed using western blotting 4,7, 10 and 15 days after the induction. miR, microRNA; NC, negative control; PPAR $\gamma$, proliferator-activated receptor $\gamma$; C/EBP- $\alpha$, CCAAT/enhancer-binding protein $\alpha$; ap2, fatty acid-binding protein; HSL, hormone-sensitive lipase.

with the negative control vector revealed that the majority of the preadipocytes $(>90 \%)$ expressed GFP, and that the miR-26b-overexpressing cells and negative control cells were morphologically similar to naive preadipocytes (Fig. 1A). RT-qPCR analysis using an miR-26b probe confirmed that the preadipocytes transduced with the miR-26b lentivirus exhibited increased expression levels of miR-26b (Fig. 1B).

Effect of miR-26b on preadipocyte differentiation. Following the addition of induction medium, human preadipocytes, originally exhibiting spindle-like features, exhibited morphological changes, becoming round and accumulating intracellular lipids. The intracellular lipid accumulation was assessed using oil red O staining, and the TG content was quantified. The miR-26b-overexpressing cells exhibited a higher intracellular lipid content, compared with the negative control cells. The number and size of the lipid droplets within the cells were increased in the miR-26b-overexpressing cells (Fig. 2A). The TG content was also increased in the miR-26b-overexpressing cells (Fig. 2B).

Effect of miR-26b on the expression of adipogenesis-associated genes. To investigate the effect of the upregulation of miR-26b on the differentiation of preadipocytes, the present study analyzed adipogenesis-associated genes using western blotting at various time-points during adipogenesis. The lentivirus-transduced-miR-26b-overexpressing preadipocytes exhibited higher protien expression levels of PPAR $\gamma$, compared with the lentivirus-transduced control cells (Fig. 3). Changes in CCAAT/enhancer-binding protein (C/EBP)- $\alpha$, fatty acid-binding protein (aP2) and hormone-sensitive lipase (HSL) during adipogenic differentiation was also investigated, which revealed similar results (Fig. 3). The analyses of these adipogenic marker genes indicated that upregulation of the 
A

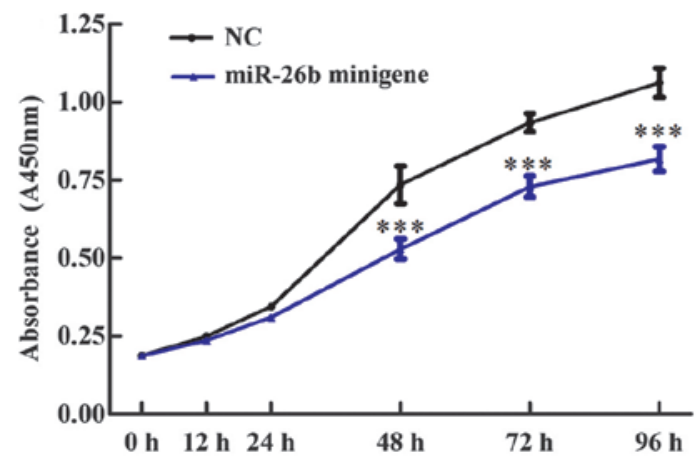

C
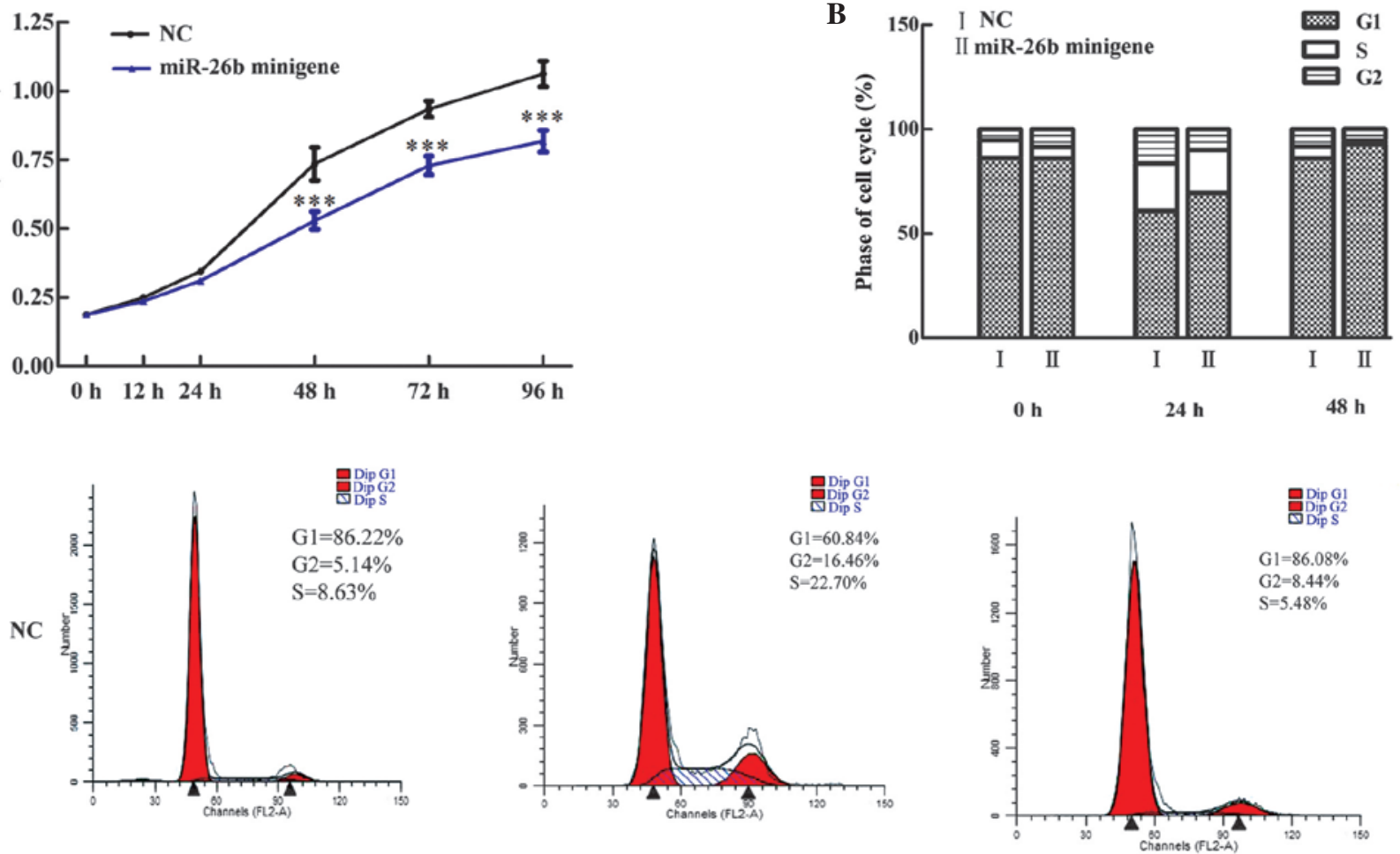

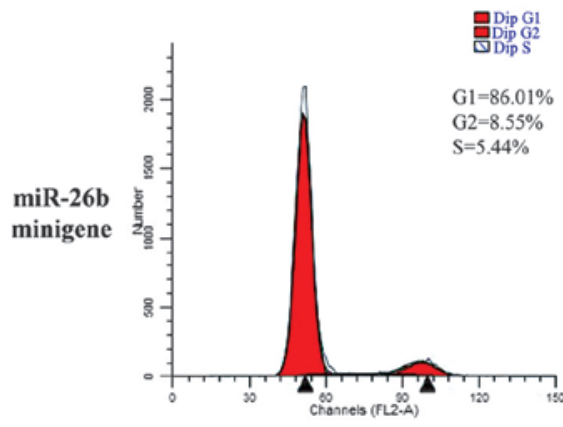

$\mathbf{0 ~ h}$
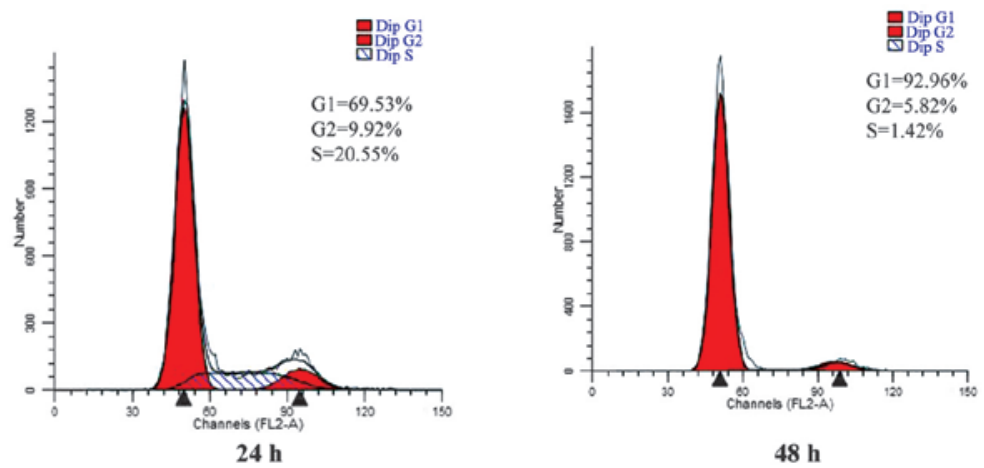

Figure 4. miR-26b attenuates the proliferation of human preadipocytes. Human preadipocytes were infected with either an miR-26b-overexpressing lentivirus (miR-26b minigene) or NC lentivirus. The rates of cell proliferation were determined using (A) Cell Counting kit-8 cell viability assays and (B and C) cell cycle analysis. A higher percentage of preadipocytes overexpressing miR-26b were present in G1 phase and a lower percentage were present in $\mathrm{S}$ phase, as compared with the negative control cells. Values are presented as the mean \pm standard deviation of three independent experiments $(* * * 2<0.001$, vs. NC). miR, microRNA; NC, negative control.

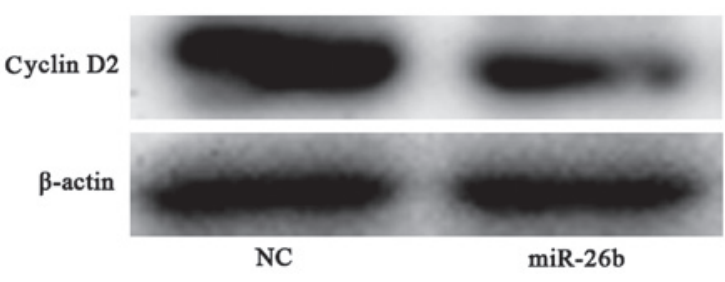

Figure 5. Expression of cyclin D2 in miR-26b minigene-transfected preadipocytes. The protein levels of cyclin D2, normalized to $\beta$-actin, were analyzed using western blotting at different time-points following miR-26b overexpression. miR, microRNA; NC, negative control.

expression of miR-26b increased the adipogenic differentiation of the preadipocytes.

Effect of miR-26b on preadipocyte proliferation. To evaluate the effects of miR-26b on the proliferation of preadipocytes, the present study detected the growth activity of preadipocytes using CCK-8 assays. Human preadipocytes overexpressing miR-26b had a decreased growth rate, compared with the negative control cells. At 12 and 24 h, no difference was observed between the absorbance of the cells overexpressing miR-26b and those transduced with the empty vectors. From $48 \mathrm{~h}$, the absorbance of the cells overexpressing miR-26b was reduced, compared with the negative control cells (Fig. 4A).

The cell cycle progression of human preadipocytes was also affected by the overexpression of miR-26b. As shown in Fig. 4B and C, flow cytometric analysis of the cell cycle distribution revealed a higher percentage of preadipocytes overexpressing miR-26b in the G1 phase and a lower percentage in the $\mathrm{S}$ phase, compared with the negative control cells, following recovery of complete medium at 24 and $48 \mathrm{~h}$. These results demonstrated that miR-26b overexpression attenuated the proliferation of human preadipocytes. In addi 
miR-26b downregulated the protein expression of cyclin D2, a key molecule in cell cycle regulation (Fig. 5).

\section{Discussion}

miR-26b is an intronic miRNA located in the carboxy-terminal domain, RNA polymerase II, polypeptide A and small phosphatase 1 , which can regulate the transcription of its host (13). In our previous study, a microarray strategy and RT-qPCR technology was used to demonstrate that miR-26b is upregulated during the differentiation of human preadipocytes to adipocytes and is associated with adipokines (14-16). Evidence is emerging that the expression of miR-26b is closely associated with obesity. For example, hsa-miR-26b is significantly associated with the number of infiltrating macrophages in abdominal subcutaneous adipose tissue (17) and the expression of mmu-miR-26b is upregulated during adipogenesis (18). Therefore, miR-26b has been reported to be differentially expressed during the differentiation of human preadipocytes, and may be a mediator of adipogenesis and proliferation in human preadipocytes.

In the present study, the effect of miR-26b on the differentiation of human preadipocytes was analyzed. Using oil red $\mathrm{O}$ staining and determining the TG content 4,10 and 15 days after differentiation, the overexpression of miR-26b in human preadipocytes was observed to increase the number and size of the lipid droplets (Fig. 2A). Overexpression of miR-26b increased the accumulation of intracellular lipids, resulting in these cells having a higher lipid content, compared with the negative control cells (Fig. 2B). These data suggested that miR-26b may be involved in adipogenesis in human preadipocytes. Studies have demonstrated that PPAR $\gamma$ and C/EBP- $\alpha$ are essential for the regulation of adipogenesis (19-21). HSL and aP2 are also involved in lipid metabolism and insulin sensitivity (22). Therefore, the present study examined the expression of these adipogenesis-associated markers in the miR-26b-overexpressing cells and found that the overexpression of miR-26b promoted their expression levels (Fig. 3). These results further indicated that miR-26b is closely associated with obesity.

Overexpression of miR-26b in the human preadipocytes also caused a decrease in cell population growth (Fig. 4A). Cell cycle analysis demonstrated an increase in the percentage of cells in the G1 phase (Fig. 4B). The increase of sub G1 cell fraction indicated that miR-26b had an inhibitory effect on the proliferation of the human preadipocytes. Apoptosis is also involved in adipose tissue mass (23), thus, the present study also assessed the effect of overexpression of miR-26b in human preadipocytes on apoptosis. However, the overexpression of miR-26b had no effect on serum deprivation-induced apoptosis (data not shown). Therefore, miR-26b was important in the proliferation of preadipocytes via cell cycle arrest in the G1 phase. To further clarify the mechanism of G1/S arrest in the preadipocytes, the expression of cell cycle-associated proteins was examined, and the overexpression of miR-26b was observed to attenuate the expression of cyclin D2 (Fig. 5). Cyclin D2 forms a complex with, and functions as a regulatory subunit of CDK4 or CDK6, the activity of which is required for the cell cycle G1/S transition. Therefore, the results of the presents study suggested that miR-26b, at least in part by targeting cyclin D2, was involved in attenuating cell proliferation via arresting the G1/S transition.
In conclusion, overexpression of miR-26b promoted adipogenesis in human preadipocytes, resulting in TG accumulation in these cells. Overexpression of miR-26b also upregulated the expression levels of the PPAR $\gamma, \mathrm{C} / \mathrm{EBP} \alpha$, aP2 and HSL adipogenesis-associated marker genes. In addition, miR-26b, at least in part by targeting cyclin D2, attenuated the proliferation of the preadipocytes via arrest of the G1/S transition. These results suggested that miR-26b contributes to the adipogenesis and proliferation of preadipocytes and may be of key importance in preventing obesity and its sequelae.

\section{Acknowledgements}

This study was supported by grants from the National Key Basic Research Program of China (grant.no. 2013CB530604), National Natural Science Foundation of China (grant. no. 81170797), Natural Science Foundation of Jiangsu Province of China (grant. no. BK2011107), Program for Innovative Research Teams of Jiangsu Province (grant. no. LJ201108) and the Nanjing Technological Development Program (grant. no. 201104013).

\section{References}

1. Haslam DW and James WP: Obesity. Lancet 366: 1197-1209, 2005.

2. Basham P and Luik J: Is the obesity epidemic exaggerated? Yes. BMJ 336: 244, 2008

3. Avram MM, Avram AS and James WD: Subcutaneous fat in normal and diseased states 3. Adipogenesis: from stem cell to fat cell. J Am Acad Dermatol 56: 472-492, 2007.

4. Hausman DB, DiGirolamo M, Bartness TJ, Hausman GJ and Martin RJ: The biology of white adipocyte proliferation. Obes Rev 2: 239-254, 2001.

5. Bushati N and Cohen SM: microRNA functions. Annu Rev Cell Dev Biol 23: 175-205, 2007.

6. Eulalio A, Huntzinger E and Izaurralde E: Getting to the root of miRNA-mediated gene silencing. Cell 132: 9-14, 2008.

7. Kim YJ, Hwang SH, Cho HH, Shin KK, Bae YC and Jung JS: MicroRNA 21 regulates the proliferation of human adipose tissue-derived mesenchymal stem cells and high-fat diet-induced obesity alters microRNA 21 expression in white adipose tissues. J Cell Physiol 227: 183-193, 2012.

8. Zhang JF, Fu WM, He ML, et al: MiR-637 maintains the balance between adipocytes and osteoblasts by directly targeting Osterix. Mol Biol Cell 22: 3955-3961, 2011.

9. Esau C, Kang X, Peralta E, et al: MicroRNA-143 regulates adipocyte differentiation. J Biol Chem 279: 52361-52365, 2004.

10. Karbiener M, Neuhold C, Opriessnig P, Prokesch A, Bogner-Strauss JG and Scheideler M: MicroRNA-30c promotes human adipocyte differentiation and co-represses PAI-1 and ALK2. RNA Biol 8: 850-860, 2011.

11. Lee EK, Lee MJ, Abdelmohsen K, et al: miR-130 suppresses adipogenesis by inhibiting peroxisome proliferator-activated receptor gamma expression. Mol Cell Biol 31: 626-638, 2011.

12. Livak KJ and Schmittgen TD: Analysis of relative gene expression data using realtime quantitative PCR and the 2(-Delta Delta C(T)) Method. Methods 25: 402-408, 2001.

13. Dill H, Linder B, Fehr A and Fischer U: Intronic miR-26b controls neuronal differentiation by repressing its host transcript, ctdsp2. Genes Dev 26: 25-30, 2012.

14. Song G, Xu G, Ji C, et al: The role of microRNA-26b in human adipocyte differentiation and proliferation. Gene 533: 481-487, 2014.

15. Xu G, Ji C, Shi C, et al: Modulation of hsa-miR-26b levels following adipokine stimulation. Mol Biol Rep 40: 3577-3582, 2013.

16. Xu G, Shi C, Ji C, et al: Expression of microRNA-26b, an obesity-related microRNA, is regulated by free fatty acids, glucose, dexamethasone and growth hormone in human adipocytes. Mol Med Rep 10: 223-228, 2014.

17. Klöting N, Berthold S, Kovacs P, et al: MicroRNA expression in human omental and subcutaneous adipose tissue. PLoS One 4: e4699, 2009. 
18. Kajimoto K, Naraba H and Iwai N: MicroRNA and 3T3-L1 pre-adipocyte differentiation. Rna 12: 1626-1632, 2006.

19. Sun F, Wang J, Pan Q, et al: Characterization of function and regulation of miR-24-1 and miR-31. Biochem Biophys Res Commun 380: 660-665, 2009.

20. Jones JR, Barrick C, Kim KA, et al: Deletion of PPARgamma in adipose tissues of mice protects against high fat diet-induced obesity and insulin resistance. Proc Natl Acad Sci USA 102: 6207-6212, 2005.

21. Zuo Y, Qiang L and Farmer SR: Activation of CCAAT/enhancer-binding protein (C/EBP) alpha expression by $\mathrm{C} / \mathrm{EBP}$ beta during adipogenesis requires a peroxisome proliferator-activated receptor-gamma-associated repression of HDAC1 at the C/ebp alpha gene promoter. J Biol Chem 281: 7960-7967, 2006.
22. Kralisch S and Fasshauer M: Adipocyte fatty acid binding protein: a novel adipokine involved in the pathogenesis of metabolic and vascular disease? Diabetologia 56: 10-21, 2013.

23. Della-Fera MA, Qian H and Baile CA: Adipocyte apoptosis in the regulation of body fat mass by leptin. Diabetes Obes Metab 3: 299-310, 2001. 